\title{
A new algorithm that generates the image of the attractor of a generalized iterated function system
}

\author{
Radu MICULESCU, Alexandru MIHAIL and Silviu-Aurelian URZICEANU
}

\begin{abstract}
We provide a new algorithm (called the grid algorithm) designed to generate the image of the attractor of a generalized iterated function system on a finite dimensional space and we compare it with the deterministic algorithm regarding generalized iterated function systems presented by P. Jaros, E. Maślanka and F. Strobin in [Algorithms generating images of attractors of generalized iterated function systems, Numer. Algorithms, 73 (2016), 477-499].

Key words and phrases: generalized infinite iterated function system (GIFS), attractor, deterministic algorithm, grid algorithm

2010 Mathematics Subject Classification: Primary 28A80; Secondary 37C70, $41 \mathrm{~A} 65,65 \mathrm{~S} 05,65 \mathrm{P} 99$
\end{abstract}

\section{Introduction}

As part of the effort to extend the classical theory of iterated function systems due to J. Hutchinson (see [2]), R. Miculescu and A. Mihail introduced the concept of generalized iterated function system (see [7] and [9]) which was obtained by considering contractions from $X^{p}$ into $X$ rather than contractions from $X$ into itself (here $(X, d)$ is a metric space and $p$ is a natural number). Sufficient conditions for the existence and uniqueness of the attractor of a generalized iterated function system (for short GIFS) $\mathcal{F}=\left((X, d),\left(f_{i}\right)_{i \in\{1,2, \ldots, L\}}\right)$, an upper bound for the Hausdorff-Pompeiu distance between the attractors of two such GIFSs, an upper bound for the Hausdorff-Pompeiu distance between the attractor of such a GIFS and an arbitrary compact set of $X$ have been provided and the continuous dependence of the attractor on the functions $f_{i}$ was proved. In the last years this concept has been intensively studied. Let us mention some lines of research regarding this subject:

In [15], F. Strobin and J. Swaczyna extended the concept of GIFS by using weaker types of generalized contractions which are similar to those introduced by F. Browder (see [1]) or J. Matkowski (see [5]). In [14], Strobin 
emphasized the fact that the set of the attractors generated by GIFSs is larger than the one generated by iterated function systems. Another related topics can be found in [4], [6], [8], [10], [11], [12], [13] and [16].

Moreover, in [3], Strobin and his collaborators provided four algorithms which generate images of attractors of GIFSs, one of them being the deterministic algorithm for GIFSs (a counterpart of the classical deterministic algorithm for iterated function systems). Note that in [3] one can also find a list of papers dealing with algorithms generating images of the attractors of iterated function systems.

In this paper we present another algorithm (called the grid algorithm) allowing to generate images of the attractors of GIFSs on finite dimensional spaces and we compare it with the deterministic algorithm for GIFSs. The deterministic algorithm for GIFSs consists in choosing a finite set of points and applying to this set each of the constitutive functions of the system obtaining in this way a new finite set of points. To each of these new points we apply again each of the constitutive functions of the system. Continuing the procedure described above we approach the attractor. The main idea of the grid algorithm is to simplify the deterministic algorithm by dividing, at each step, the space that we are working with in small pieces and to choose for each such piece just one point.

\section{Preliminaries}

Given a metric space $(X, d)$, we adopt the following notation:

$$
P_{c p}(X) \stackrel{\text { not }}{=}\{K \subseteq X \mid K \text { is non-empty and compact }\} .
$$

For $K_{1}, K_{2} \in P_{c p}(X)$, we consider

$$
d\left(K_{1}, K_{2}\right) \stackrel{\text { def }}{=} \sup _{x \in K_{1}} d\left(x, K_{2}\right),
$$

where $d\left(x, K_{2}\right) \stackrel{\text { def }}{=} \inf _{y \in K_{2}} d(x, y)$.

The function $h: P_{c p}(X) \times P_{c p}(X) \rightarrow[0, \infty)$ given by

$$
h\left(K_{1}, K_{2}\right)=\max \left\{d\left(K_{1}, K_{2}\right), d\left(K_{2}, K_{1}\right)\right\},
$$

for every $K_{1}, K_{2} \in P_{c p}(X)$, turns out to be a metric which is called the Hausdorff-Pompeiu metric. 
If $(X, d)$ is complete, then $\left(P_{c p}(X), h\right)$ is complete.

Given a metric space $(X, d)$ and $p \in \mathbb{N}^{*}$, by $X^{p}$ we denote the Cartesian product of $p$ copies of $X$. We endow $X^{p}$ with the maximum metric $d_{\max }$ defined by

$$
d_{\max }\left(\left(x_{1}, \ldots, x_{p}\right),\left(y_{1}, \ldots, y_{p}\right)\right)=\max \left\{d\left(x_{1}, y_{1}\right), \ldots, d\left(x_{p}, y_{p}\right)\right\}
$$

for all $\left(x_{1}, \ldots, x_{p}\right),\left(y_{1}, \ldots, y_{p}\right) \in X^{p}$.

Definition 2.1. A generalized iterated function system (of order $p$ ) is a pair $\mathcal{F}=\left((X, d),\left(f_{i}\right)_{i \in\{1,2, \ldots, L\}}\right)$, where $(X, d)$ is a metric space, $p, L \in \mathbb{N}^{*}$ and $f_{i}: X^{p} \rightarrow X$ is contraction for each $i \in\{1, \ldots, L\}$. The function $F_{\mathcal{F}}:\left(P_{c p}(X)\right)^{p} \rightarrow P_{c p}(X)$, described by

$$
F_{\mathcal{F}}\left(K_{1}, \ldots, K_{p}\right)=\underset{i \in\{1, \ldots, L\}}{\cup} f_{i}\left(K_{1} \times \ldots \times K_{p}\right),
$$

for all $K_{1}, \ldots, K_{p} \in P_{c p}(X)$, is called the fractal operator associated to $\mathcal{F}$.

We shall use the abbreviation GIFS for a generalized iterated function system.

Theorem 2.2 (see Theorem 3.9 from [9]). Given a complete metric space $(X, d)$ and a GIFS $\mathcal{F}=\left((X, d),\left(f_{i}\right)_{i \in\{1, \ldots, L\}}\right)$ of order $p$, there exists a unique $A_{\mathcal{F}} \in P_{c p}(X)$ such that

$$
F_{\mathcal{F}}\left(A_{\mathcal{F}}, \ldots, A_{\mathcal{F}}\right)=A_{\mathcal{F}} .
$$

In addition, for every $K_{1}, \ldots, K_{p} \in P_{c p}(X)$, the sequence $\left(K_{n}\right)_{n}$ defined by

$$
K_{n+p}=F_{\mathcal{F}}\left(K_{n}, \ldots, K_{n+p-1}\right),
$$

for every $n \in \mathbb{N}^{*}$, converges, with respect to the Hausdorff-Pompeiu metric, to $A_{\mathcal{F}}$.

Definition 2.3. In the framework of the above theorem, the set $A_{\mathcal{F}}$ is called the fractal generated by $\mathcal{F}$.

Remark 2.4 (see Remark 12 from [3]). In the framework of the above definition, the function $\mathcal{G}_{\mathcal{F}}: P_{c p}(X) \rightarrow P_{c p}(X)$, described by

$$
\mathcal{G}_{\mathcal{F}}(K)=F_{\mathcal{F}}(K, \ldots, K)=\underset{i \in\{1, \ldots, L\}}{\cup} f_{i}(K \times \ldots \times K),
$$


for all $K \in P_{c p}(X)$, is a contraction on the complete metric space $\left(P_{c p}(X), h\right)$ since it has the Lipschitz constant less of equal to $\max \left\{\operatorname{lip}\left(f_{1}\right), \ldots, \operatorname{lip}\left(f_{L}\right)\right\}<$ 1.

\section{The presentation of the algorithms}

For $\left(x_{1}, \ldots, x_{M}\right) \in \mathbb{R}^{M}$, we shall use the following notation:

$$
\left[\left(x_{1}, \ldots, x_{M}\right)\right]=\left(\left[x_{1}\right], \ldots,\left[x_{M}\right]\right)
$$

where $[x]$ designates the greatest integer less than or equal to the real number $x$.

In the sequel, without loss of generality,

$$
\mathcal{F}=\left(\left([0, D]^{M}, d\right),\left\{f_{1}, \ldots, f_{L}\right\}\right),
$$

where $L, M \in \mathbb{N}$ and $d$ is the euclidean distance in $\mathbb{R}^{M}$, will be a generalized iterated function system of order $p \geq 2\left(\right.$ so $f_{i}:\left([0, D]^{M}\right)^{p} \rightarrow[0, D]^{M}$ for every $i \in\{1, \ldots, L\})$.

We shall use the following notation:

- $\max \left\{\operatorname{lip}\left(f_{1}\right), \ldots, \operatorname{lip}\left(f_{L}\right)\right\} \stackrel{\text { not }}{=} C<1$

- $\beta=p M$.

We also consider the following functions:

- $F_{\mathcal{F}}:\left(P_{c p}\left([0, D]^{M}\right)\right)^{p} \rightarrow P_{c p}\left([0, D]^{M}\right)$ described by

$$
F_{\mathcal{F}}\left(K_{1}, \ldots, K_{p}\right)=f_{1}\left(K_{1} \times \ldots \times K_{p}\right) \cup \ldots \cup f_{L}\left(K_{1} \times \ldots \times K_{p}\right),
$$

for all $K_{1}, \ldots, K_{p} \in P_{c p}\left([0, D]^{M}\right)$

- $\mathcal{G}_{\mathcal{F}}: P_{c p}\left([0, D]^{M}\right) \rightarrow P_{c p}\left([0, D]^{M}\right)$ described by

$$
\mathcal{G}_{\mathcal{F}}(K)=F_{\mathcal{F}}(K, \ldots, K),
$$

for every $K \in P_{c p}\left([0, D]^{M}\right)$.

- $\left(n_{k}\right)_{k \in \mathbb{N}^{*}}$ a sequence of natural numbers.

For a finite set $K_{0} \in P_{c p}\left([0, D]^{M}\right)$, we shall use the following notations:

$$
A_{k} \stackrel{\text { not }}{=} \mathcal{G}_{\mathcal{F}}^{[k]}\left(K_{0}\right)
$$

where $k \in \mathbb{N}$ 


$$
\tilde{A}_{k} \stackrel{n o t}{=}\left\{\frac{D}{n_{k}}\left[\frac{n_{k}}{D} f_{l}\left(u_{1}, \ldots, u_{p}\right)\right] \mid u_{1}, \ldots, u_{p} \in \tilde{A}_{k-1}, l \in\{1, \ldots, L\}\right\}
$$

where $k \in \mathbb{N}^{*}$ and $\tilde{A_{0}}=K_{0}$

$$
\frac{D \sqrt{M}}{n_{k}} \stackrel{n o t}{=} \varepsilon_{k}
$$

where $k \in \mathbb{N}$.

Let us recall the pseudocode for the deterministic algorithm for a GIFS (see [3])

\section{Pseudocode for the deterministic algorithm for a GIFS}

Read initially defined objects: constants: $L, M$, finite set, $m$ natural number: $K_{0} \in P_{c p}\left([0, D]^{M}\right)$, mappings: $f_{1}, \ldots, f_{L}$, variables: $k, D_{0}$.

Initial values: $D_{0}:=K_{0}$.

For $k$ from 1 to $m-1$

$$
\begin{aligned}
D_{1} & :=\mathcal{G}_{\mathcal{F}}\left(D_{0}\right) \\
D_{0} & :=D_{1} .
\end{aligned}
$$

Print $D_{m}$.

Now let us present the pseudocode for our new algorithm.

\section{Pseudocode for the grid algorithm for a GIFS}

Read initially defined objects: constant: $L, M$, finite set, $m$ natural number: $K_{0} \in P_{c p}\left([0, D]^{M}\right)$, mappings: $f_{1}, \ldots, f_{L}$, sequence: $\left(n_{k}\right)_{k}$, variables: $k, D_{0}$.

Initial values: $D_{0}:=K_{0}$.

For $k$ from 1 to $m-1$

$$
\begin{aligned}
& D_{1}:=\left\{\frac{D}{n_{k}}\left[\frac{n_{k}}{D} f_{l}\left(u_{1}, \ldots, u_{p}\right)\right] \mid u_{1}, \ldots, u_{p} \in D_{0}, l \in\{1, \ldots, L\}\right\} \\
& D_{0}:=D_{1} .
\end{aligned}
$$

Print $D_{m}$. 


\section{The complexity of the algorithms}

By $x_{k}$ we denote the number of points computed at the step $k$ of the deterministic algorithm and by $y_{k}$ the number of points computed up to the step $k$ of the grid algorithm.

A. The case of the deterministic algorithm

We have $x_{k+1} \leq L\left(x_{k}\right)^{p}$, so, with the notation $z_{k} \stackrel{\text { not }}{=} \ln x_{k}$, we obtain $z_{k+1} \leq \ln L+p z_{k}$ for every $k \in \mathbb{N}$. Therefore $z_{k} \leq \frac{p^{k}-1}{p-1} \ln L+p^{k} z_{0}$, i.e.

$$
x_{k} \leq \frac{1}{L^{\frac{1}{p-1}}}\left(x_{0} L^{\frac{1}{p-1}}\right)^{p^{k}}
$$

for every $k \in \mathbb{N}$.

Note that, according to Remark 2.4, we have $h\left(A_{k}, A_{\mathcal{F}}\right) \leq \frac{h\left(A_{0}, A_{1}\right)}{1-C} C^{k}$ for every $k \in \mathbb{N}$. Therefore, in order to be sure that $A_{k}$ approximates the attractor $A_{\mathcal{F}}$ with accuracy $\varepsilon \frac{h\left(A_{0}, A_{1}\right)}{1-C}$, we need $k>\log _{C^{-1}}\left(\varepsilon^{-1}\right)$. Hence, based on (1), the quantity $\frac{1}{L^{\frac{1}{p-1}}}\left(x_{0} L^{\frac{1}{p-1}}\right)^{p^{\log _{C^{-1}}\left(\varepsilon^{-1}\right)}}=\frac{1}{L^{\frac{1}{p-1}}}\left(x_{0} L^{\frac{1}{p-1}}\right)^{\left(\frac{1}{\varepsilon}\right)^{\log _{C^{-1}}(p)}}$ describes the number of points that we have to compute in order to be sure that $A_{k}$ is an approximation of $A_{\mathcal{F}}$ with an error less than $\varepsilon \frac{h\left(A_{0}, A_{1}\right)}{1-C}$.

Conclusion: The complexity of the deterministic algorithm is described by the function $\mathcal{C}_{c}:(0, \infty) \rightarrow \mathbb{R}$ given by

$$
\mathcal{C}_{c}(\varepsilon)=\left(x_{0} L^{\frac{1}{p-1}}\right)^{\left(\frac{1}{\varepsilon}\right)^{\log _{\frac{1}{C}}(p)}},
$$

for every $\varepsilon>0$.

B. The case of the grid algorithm

Remark 4.1. Since $y_{k+1} \leq L\left(n_{k}\right)^{\beta}$ for every $k \in \mathbb{N}^{*}$, up to the step $N$, we have to compute $L \sum_{k=1}^{N}\left(n_{k}\right)^{\beta}=L(D \sqrt{M})^{\beta} \sum_{k=1}^{N}\left(\frac{1}{\varepsilon_{k}}\right)^{\beta}$ points.

Remark 4.2. We have

$$
h\left(\tilde{A}_{k}, \mathcal{G}_{\mathcal{F}}\left(\tilde{A}_{k-1}\right)\right) \leq \varepsilon_{k},
$$


for every $k \in \mathbb{N}^{*}$.

Remark 4.3. We have

$$
h\left(\tilde{A}_{0}, A_{\mathcal{F}}\right) \leq \operatorname{diam}\left([0, D]^{M}\right)=D \sqrt{M} .
$$

As the inequality

$$
\begin{gathered}
h\left(\tilde{A}_{k}, A_{\mathcal{F}}\right) \leq h\left(\tilde{A}_{k}, \mathcal{G}_{\mathcal{F}}\left(\tilde{A}_{k-1}\right)\right)+h\left(\mathcal{G}_{\mathcal{F}}\left(\tilde{A}_{k-1}\right), \mathcal{G}_{\mathcal{F}}\left(A_{\mathcal{F}}\right)\right) \leq \\
\stackrel{\text { Remarks 2.4 and 4.2 }}{\leq} \varepsilon_{k}+\operatorname{Ch}\left(\tilde{A}_{k-1}, A_{\mathcal{F}}\right),
\end{gathered}
$$

is valid for every $k \in \mathbb{N}^{*}$, we get

$$
h\left(\tilde{A}_{k}, A_{\mathcal{F}}\right) \leq \varepsilon_{k}+C \varepsilon_{k-1}+C^{2} \varepsilon_{k-2}+\ldots+C^{k-2} \varepsilon_{2}+C^{k-1} \varepsilon_{1}+C^{k} h\left(\tilde{A}_{0}, A_{\mathcal{F}}\right),
$$

so, taking into account Remark 4.3, we obtain

$$
h\left(\tilde{A}_{k}, A_{\mathcal{F}}\right) \leq \varepsilon_{k}+C \varepsilon_{k-1}+C^{2} \varepsilon_{k-2}+\ldots+C^{k-2} \varepsilon_{2}+C^{k-1} \varepsilon_{1}+C^{k} D \sqrt{M},
$$

for every $k \in \mathbb{N}^{*}$. Consequently, we arrive to the following problem: given a fixed natural number $N$ and $\varepsilon>0$ such that $\frac{\varepsilon}{C^{N}}-D \sqrt{M}>0$, find the minimum of the function $f:[0, \infty)^{N} \rightarrow[0, \infty)$, given by

$$
f\left(\varepsilon_{1}, \ldots, \varepsilon_{N}\right)=\sum_{k=1}^{N}\left(\frac{1}{\varepsilon_{k}}\right)^{\beta},
$$

for every $\varepsilon_{1}, \ldots, \varepsilon_{N} \in[0, \infty)$, with the constraint

$$
\varepsilon_{N}+C \varepsilon_{N-1}+C^{2} \varepsilon_{N-2}+\ldots+C^{N-2} \varepsilon_{2}+C^{N-1} \varepsilon_{1}+C^{N} D \sqrt{M}=\varepsilon .
$$

We adopt the following notations:

- $t \stackrel{\text { not }}{=} C^{-\frac{\beta}{\beta+1} N}-1$

- $K_{1} \stackrel{\text { not }}{=} \frac{C^{\frac{1}{\beta+1}}-C}{C^{\frac{1}{\beta+1}}}=1-C^{\frac{\beta}{\beta+1}}$

- $K_{2} \stackrel{\text { not }}{=} K_{1}^{-\beta-1}$

- $K_{3} \stackrel{\text { not }}{=} K_{2} \varepsilon^{-\beta}$

- $a \stackrel{\text { not }}{=} \frac{D \sqrt{M}}{\varepsilon}$ 
- $y \stackrel{\text { not }}{=} \frac{1}{C^{N}}$.

Since we are going to use the method of Lagrange multipliers, we consider the function $F=f+\lambda g$, where $\lambda \in \mathbb{R}$ and the function $g:[0, \infty)^{N} \rightarrow[0, \infty)$ is given by

$$
g\left(\varepsilon_{1}, \ldots, \varepsilon_{N}\right)=\varepsilon_{N}+C \varepsilon_{N-1}+\ldots+C^{N-2} \varepsilon_{2}+C^{N-1} \varepsilon_{1}+C^{N} D \sqrt{M}-\varepsilon,
$$

for every $\varepsilon_{1}, \ldots, \varepsilon_{N} \in[0, \infty)$. The equation $\frac{\partial F}{\partial \varepsilon_{k}}=0$, i.e. $-\beta\left(\varepsilon_{k}\right)^{-\beta-1}+$ $\lambda C^{N-k}=0$, has the solution

$$
\varepsilon_{k}^{0}=k_{N} C^{\frac{k}{\beta+1}}
$$

for every $k$, where $k_{N}=\frac{1}{C^{\frac{N}{\beta+1}}}\left(\frac{\beta}{\lambda}\right)^{\frac{1}{\beta+1}}$. As $g\left(\varepsilon_{1}^{0}, \ldots, \varepsilon_{N}^{0}\right)=0$, we get

$k_{N}\left(C^{\frac{N}{\beta+1}}+C^{1+\frac{N-1}{\beta+1}}+C^{2+\frac{N-2}{\beta+1}}+\ldots+C^{N-2+\frac{2}{\beta+1}}+C^{N-1+\frac{1}{\beta+1}}\right)=\varepsilon-C^{N} D \sqrt{M}$,

i.e.

$$
k_{N} C^{\frac{N}{\beta+1}}\left(1+C^{\frac{\beta}{\beta+1}}+C^{2 \frac{\beta}{\beta+1}}+\ldots+C^{(N-2) \frac{\beta}{\beta+1}}+C^{(N-1) \frac{\beta}{\beta+1}}\right)=\varepsilon-C^{N} D \sqrt{M}
$$

so $k_{N} C^{\frac{N}{\beta+1}} \frac{\left(C^{\frac{\beta}{\beta+1}}\right)^{N}-1}{C^{\frac{\beta}{\beta+1}}-1}=\varepsilon-C^{N} D \sqrt{M}$, which implies $k_{N} \frac{C^{N}-C^{\frac{N}{\beta+1}}}{C-C^{\frac{1}{\beta+1}}}=\frac{\varepsilon-C^{N} D \sqrt{M}}{C^{\frac{1}{\beta+1}}}$.

The last equality takes the form $k_{N} \frac{C^{-\frac{\beta}{\beta+1}}{ }^{N}-1}{C^{\frac{1}{\beta+1}}-C}=\frac{\frac{\varepsilon}{C^{N}}-D \sqrt{M}}{C^{\frac{1}{\beta+1}}}$. Thus we obtain

$$
k_{N}=\frac{K_{1}}{t}\left(\frac{\varepsilon}{C^{N}}-D \sqrt{M}\right)
$$

We have

$$
\begin{gathered}
f\left(\varepsilon_{1}^{0}, \ldots, \varepsilon_{N}^{0}\right)=\sum_{k=1}^{N}\left(\varepsilon_{k}^{0}\right)^{-\beta} \stackrel{(1)}{=}\left(k_{N}\right)^{-\beta} \sum_{k=1}^{N} C^{-\frac{\beta}{\beta+1} k}=\left(k_{N}\right)^{-\beta} C^{-\frac{\beta}{\beta+1}} \frac{\left(C^{-\frac{\beta}{\beta+1}}\right)^{N}-1}{C^{-\frac{\beta}{\beta+1}}-1}= \\
\stackrel{(2)}{=} t^{\beta} K_{1}^{-\beta}\left(\frac{\varepsilon}{C^{N}}-D \sqrt{M}\right)^{-\beta} \frac{t}{1-C^{\frac{\beta}{\beta+1}}}=t^{\beta+1}\left(\frac{\varepsilon}{C^{N}}-D \sqrt{M}\right)^{-\beta} \frac{K_{1}^{-\beta}}{K_{1}}= \\
=t^{\beta+1}\left(\frac{\varepsilon}{C^{N}}-D \sqrt{M}\right)^{-\beta} K_{1}^{-\beta-1}=K_{2}\left(\frac{\varepsilon}{C^{N}}-D \sqrt{M}\right)^{-\beta}\left(C^{-\frac{\beta}{\beta+1}} N-1\right)^{\beta+1} .
\end{gathered}
$$

Therefore, the last equality can be written as

$$
f\left(\varepsilon_{1}^{0}, \ldots, \varepsilon_{N}^{0}\right)=K_{3}\left(y^{\frac{\beta}{\beta+1}}-1\right)^{\beta+1}(y-a)^{-\beta} .
$$


As the right hand side of (3) gives us the optimal number of points that we have to compute, after $N$ steps, in order to approximate $A_{\mathcal{F}}$ by $\tilde{A}_{k}$ with an error not greater than $\varepsilon$, we need to find the minimum value of the function $h:(a, \infty) \rightarrow \mathbb{R}$ given by

$$
h(y)=K_{3}\left(y^{\frac{\beta}{\beta+1}}-1\right)^{\beta+1}(y-a)^{-\beta},
$$

for every $y \in(a, \infty)$. One can easily see that

$$
h^{\prime}(y)=K_{3} \beta\left(y^{\frac{\beta}{\beta+1}}-1\right)^{\beta}(y-a)^{-\beta-1}\left(1-a y^{-\frac{1}{\beta+1}}\right),
$$

for every $y \in(a, \infty)$. As we can suppose that $a>1$ (since we are interested in the case when $\varepsilon$ is small), $\lim _{y \rightarrow \infty} h(y)=K_{3}$ and $\lim _{\substack{y \rightarrow a \\ y>a}} h(y)=\infty$, we conclude that $h$ attains its minimum at $a^{\beta+1}$ and the value of the minimum is

$$
\begin{gathered}
h\left(a^{\beta+1}\right)=K_{3}\left(a^{\beta}-1\right)^{\beta+1}\left(a^{\beta+1}-a\right)^{-\beta}= \\
=K_{3} \frac{a^{\beta}-1}{a^{\beta}}=\varepsilon^{-\beta}\left(1-C^{\frac{\beta}{\beta+1}}\right)^{-\beta-1} \frac{\left(\frac{D \sqrt{M}}{\varepsilon}\right)^{\beta}-1}{\left(\frac{D \sqrt{M}}{\varepsilon}\right)^{\beta}},
\end{gathered}
$$

so

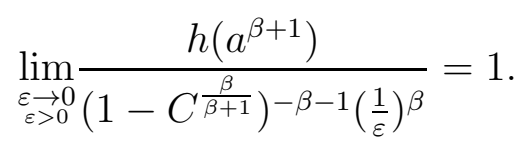

Conclusion: The complexity of the grid algorithm is described by the function $\mathcal{C}_{g}:(0, \infty) \rightarrow \mathbb{R}$ given by

$$
\mathcal{C}_{g}(\varepsilon)=\left(1-C^{\frac{\beta}{\beta+1}}\right)^{-\beta-1}\left(\frac{1}{\varepsilon}\right)^{p M},
$$

for every $\varepsilon>0$.

In the final of this section we mention that (in order to avoid very complicated computations) we did not pay attention to the fact that the best values of $n_{k}$ and $N$ that we obtained (namely $\frac{D \sqrt{M}}{\varepsilon_{k}^{0}}$ and $(\beta+1) \frac{\ln \left(\frac{\varepsilon}{D \sqrt{M}}\right)}{\ln (C)}$ ) may not be integers. In reality we could work with $n_{k}=\left[\frac{D \sqrt{M}}{\varepsilon_{k}^{0}}\right]+1$ and $N=\left[(\beta+1) \frac{\ln \left(\frac{\varepsilon}{D \sqrt{M}}\right)}{\ln (C)}\right]+1$ without a significant change. 


\section{Final remarks}

Remark 5.1. We have

$$
\lim _{\substack{\varepsilon \rightarrow 0 \\ \varepsilon>0}} \frac{\mathcal{C}_{g}(\varepsilon)}{\mathcal{C}_{c}(\varepsilon)}=\lim _{\substack{\varepsilon \rightarrow 0 \\ \varepsilon>0}} \frac{\left(1-C^{\frac{\beta}{\beta+1}}\right)^{-\beta-1}\left(\frac{1}{\varepsilon}\right)^{p M}}{\left(x_{0} L^{\frac{1}{p-1}}\right)^{\left(\frac{1}{\varepsilon}\right)^{\log } \frac{1}{C}(p)}}=0,
$$

so the grid algorithm is more efficient than the deterministic algorithm.

Remark 5.2. As $\left|u-\left[u+\frac{1}{2}\right]\right| \leq \frac{1}{2}$, we can improve our grid algorithm (which is based on the inequality $|u-[u]|<1$ ) in the following way:

\section{Pseudocode for the improved grid algorithm for GIFS}

Read initially defined objects: constant: $L, M$, finite set, $m$ natural number: $K_{0} \in P_{c p}\left([0, D]^{M}\right)$, mappings: $f_{1}, \ldots, f_{L}$, sequence: $\left(n_{k}\right)_{k}$, variables: $k, D_{0}$.

Initial values: $D_{0}:=K_{0}$.

For $k$ from 1 to $m-1$

$$
\begin{aligned}
& D_{1}:=\left\{\frac{D}{n_{k}}\left[\frac{n_{k}}{D} f_{l}\left(u_{1}, \ldots, u_{p}\right)+\frac{1}{2}\right] \mid u_{1}, \ldots, u_{p} \in D_{0}, l \in\{1, \ldots, L\}\right\} \\
& D_{0}:=D_{1} .
\end{aligned}
$$

Print $D_{m}$.

Remark 5.3. On the one hand, repeating the arguments used in IV, A, for the case of an iterated function system (i.e. $p=1$ ), we obtain that the complexity of the corresponding algorithm is described by the function $\mathcal{C}:(0, \infty) \rightarrow \mathbb{R}$ given by

$$
\mathcal{C}(\varepsilon)=\left(\frac{1}{\varepsilon}\right)^{\frac{\ln L}{\ln \frac{1}{C}}}
$$

for every $\varepsilon>0$, so $C$ is involved at the exponent of $\frac{1}{\varepsilon}$. We stress upon the fact that since $\lim _{\substack{C \rightarrow 1 \\ C<1}} \frac{1}{\ln \frac{1}{C}}=\infty$, the closer is $C$ to 1 , the bigger is the number of points that we have to compute in order to approximate the attractor with an error less that $\varepsilon$.

On the other hand, in the rule that gives $\mathcal{C}_{g}(\varepsilon)$ the constant $C$ is involved only in the coefficient $\left(1-C^{\frac{\beta}{\beta+1}}\right)^{-\beta-1}$.

Moreover, note that $\lim _{\substack{C \rightarrow 1 \\ C<1}} \frac{\mathcal{C}_{g}(\varepsilon)}{\mathcal{C}_{c}(\varepsilon)}=0$ for each $\varepsilon \in(0,1)$. 


\section{Examples}

In section IV we compared the algorithms with respect to a fixed preassigned error. In this section our goal is to get an optimal image (with respect to the computer that we worked with) for three examples. For this reason we chose a version of the grid algorithm for which $n_{k}=k^{2}$, the error being less than $D \sqrt{M}\left(\frac{1}{n^{2}}+C \frac{1}{(n-1)^{2}}+C^{2} \frac{1}{(n-2)^{2}}+\ldots+C^{n}\right)$, where $n$ is the number of steps and $C$ is the contraction constant of the system.

A.

Consider the GIFS $\mathcal{F}=\left(\left([0,1]^{2}, d\right),\left\{f_{1}, f_{2}, f_{3}\right\}\right)$, where, for $x=\left(x_{1}, y_{1}\right)$ and $y=\left(x_{2}, y_{2}\right)$, we have

$$
\begin{gathered}
f_{1}(x, y)=\left(0.2 x_{1}+0.2 y_{2} ; 0.2 x_{2}+0.1 y_{2}\right) \\
f_{2}(x, y)=\left(0.15 x_{1}+0.07 x_{2}+0.4 ; 0.15 y_{1}+0.07 y_{2}\right) .
\end{gathered}
$$

and

$$
f_{3}(x, y)=\left(0.15 y_{1}+0.07 x_{2} ; 0.15 x_{1}+0.07 y_{2}+0.04\right) .
$$

Using the deterministic algorithm we get the image indicated in figure 1 and using the grid algorithm we get the image in figure 2 .

The deterministic algorithm run 4 steps in 10 seconds, while the grid algorithm 8 steps in less that 10 seconds.

Figure 1 has approximately $3^{2^{4}}=43046721$ points, while figure 2 comprises around 20000 points.

Remark 6.1. If we allow the deterministic algorithm to run 5 steps it needs about 90 minutes and we get a very similar image with the one in figure 2. 


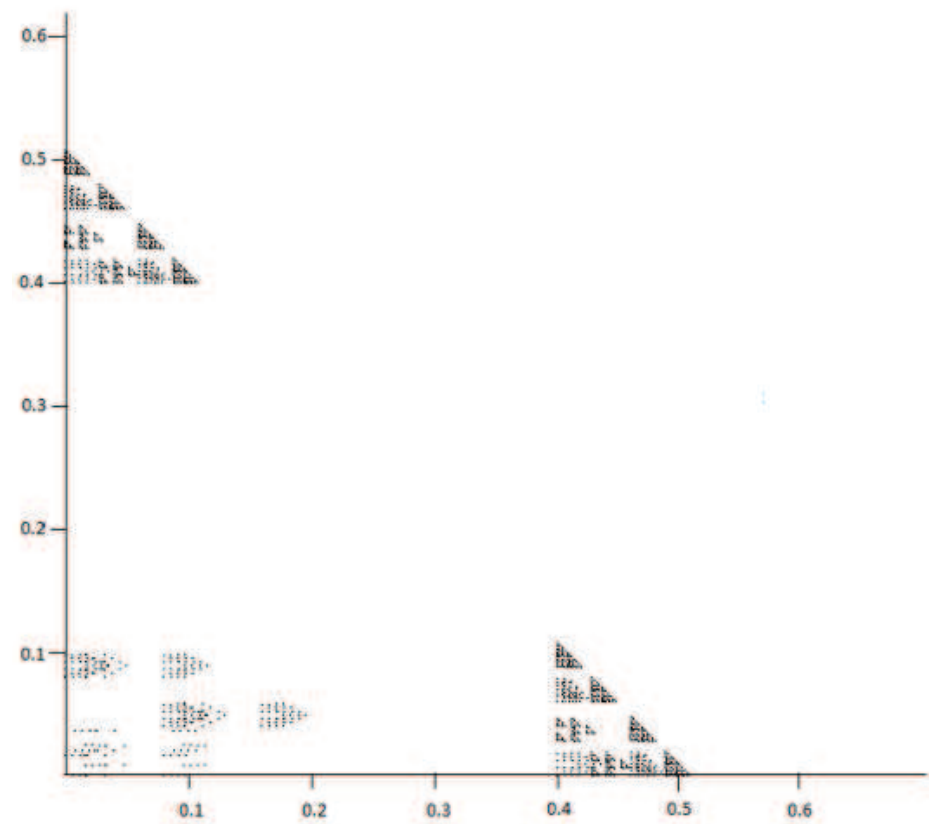

Figure 1

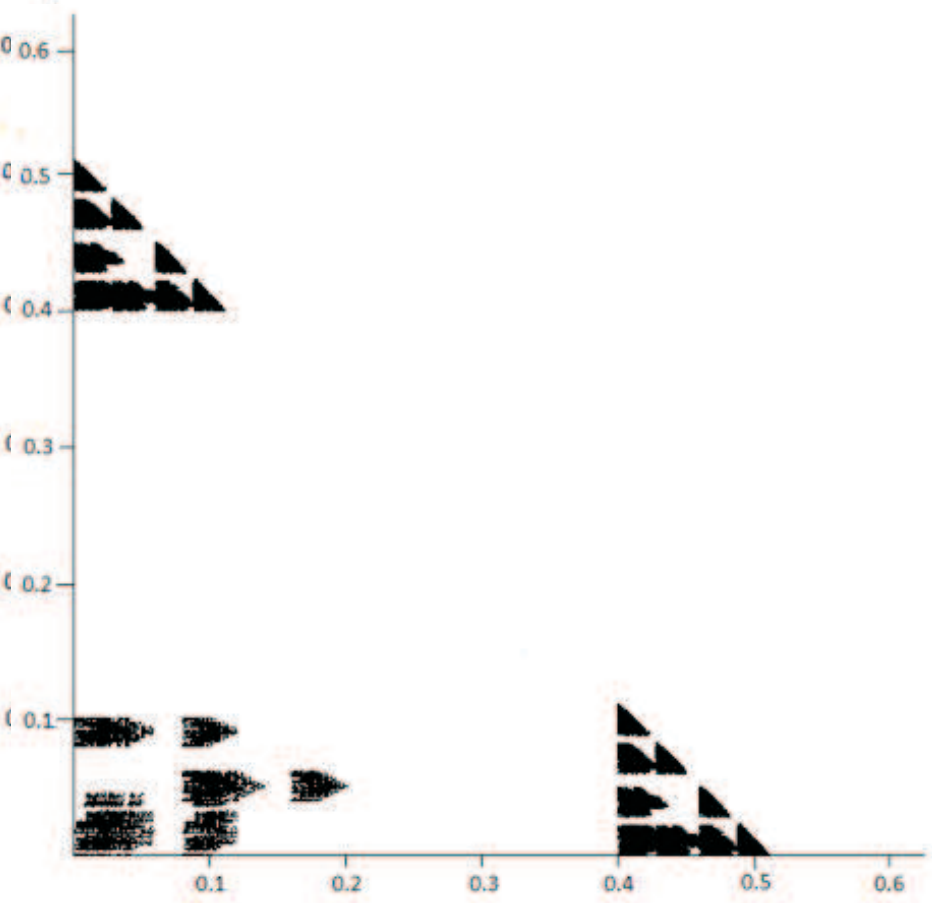

Figure 2 
B.

Consider the GIFS $\mathcal{F}=\left(\left([0,1]^{2}, d\right),\left\{f_{1}, f_{2}\right\}\right)$, where, for $x=\left(x_{1}, y_{1}\right)$ and $y=\left(x_{2}, y_{2}\right)$, we have

$$
f_{1}(x, y)=\left(0.1 x_{1}+0.16 y_{1}-0.01 x_{2}+0.3 y_{2} ;-0.05 y_{1}+0.15 x_{2}+0.15 y_{2}\right)
$$

and

$f_{2}(x, y)=\left(0.09 x_{1}-0.1 y_{1}-0.15 x_{2}+0.14 y_{2}+0.4 ; 0.14 x_{1}+0.14 y_{1}+0.14 x_{2}+0.04\right)$

The deterministic algorithm yields the image in figure 3 and the grid algorithm produces the image in figure 4.

The deterministic algorithm needed 20 seconds to run 5 steps, while the grid algorithm needed about 10 minutes to run 14 steps.

Figure 3 consists of about $2^{2^{5}}=4294967296$ points, while figure 4 is built up using around 2000000 p oints.

Remark 6.2. With the aid of the computer that we utilized, the deterministic algorithm would need 42 days to run 6 steps.

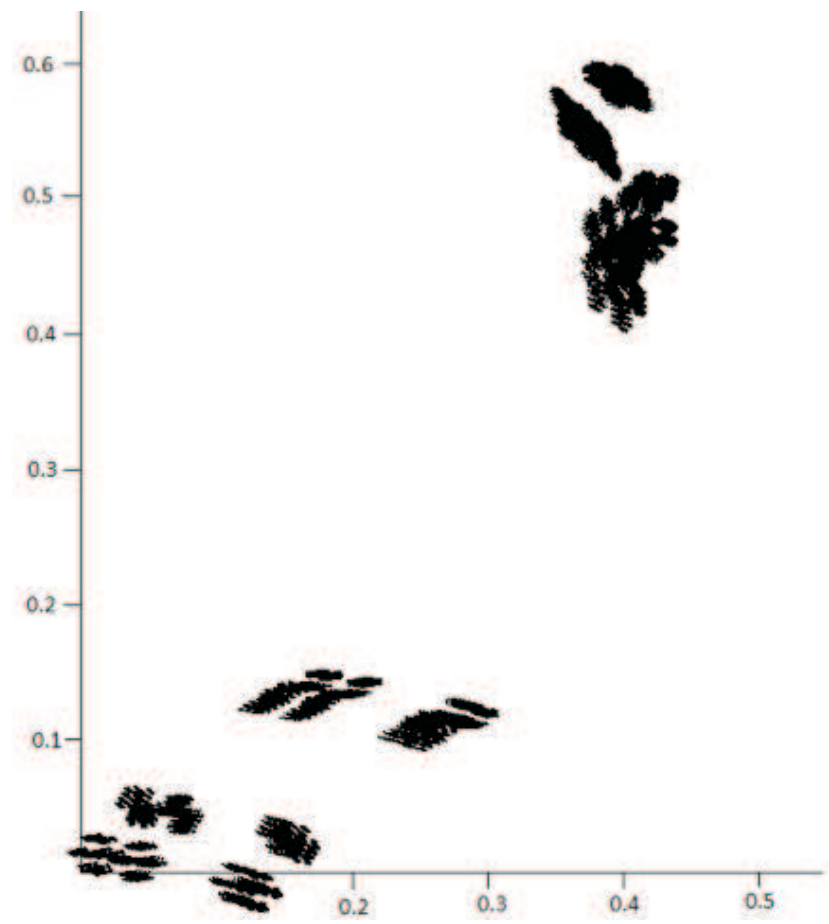

Figure 3 


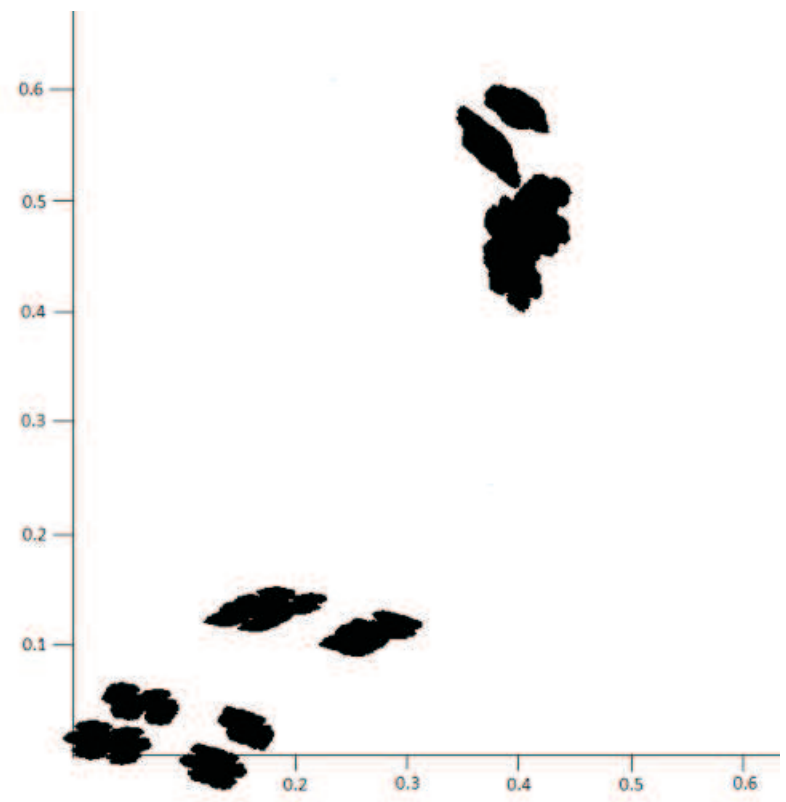

Figure 4

C.

Consider the GIFS $\mathcal{F}=\left(\left([0,1]^{2}, d\right),\left\{f_{1}, f_{2}\right\}\right)$, where, for $x=\left(x_{1}, y_{1}\right)$ and $y=\left(x_{2}, y_{2}\right)$, we have

$$
f_{1}(x, y)=\left(0.5 x_{1}-0.5 y_{1}+0.001 x_{2}+0.45 ; 0.5 x_{1}+0.5 y_{1}+0.001 y_{2}-0.05\right)
$$

and

$$
f_{2}(x, y)=\left(0.2 x_{1}+0.01 x_{2}+0.14 y_{2}+0.147 ; 0.2 y_{1}+0.01 y_{2}+0.105\right) .
$$

The image in figure 5 indicates what we get running the deterministic algorithm and the image in figure 6 what we obtain using the grid algorithm.

Both algorithms ran about 2 minutes, the deterministic one running 5 steps, while the grid one 22 steps.

Figure 5 consists of about $2^{2^{5}}=4294967296$ points, while figure 6 is made up of circa 217800 points.

Remark 6.3. Even though the number of points making up figure 5 is considerably bigger that the number of points building up figure 6, one can observe that the grid algorithm produces a much better approximation of the attractor. 


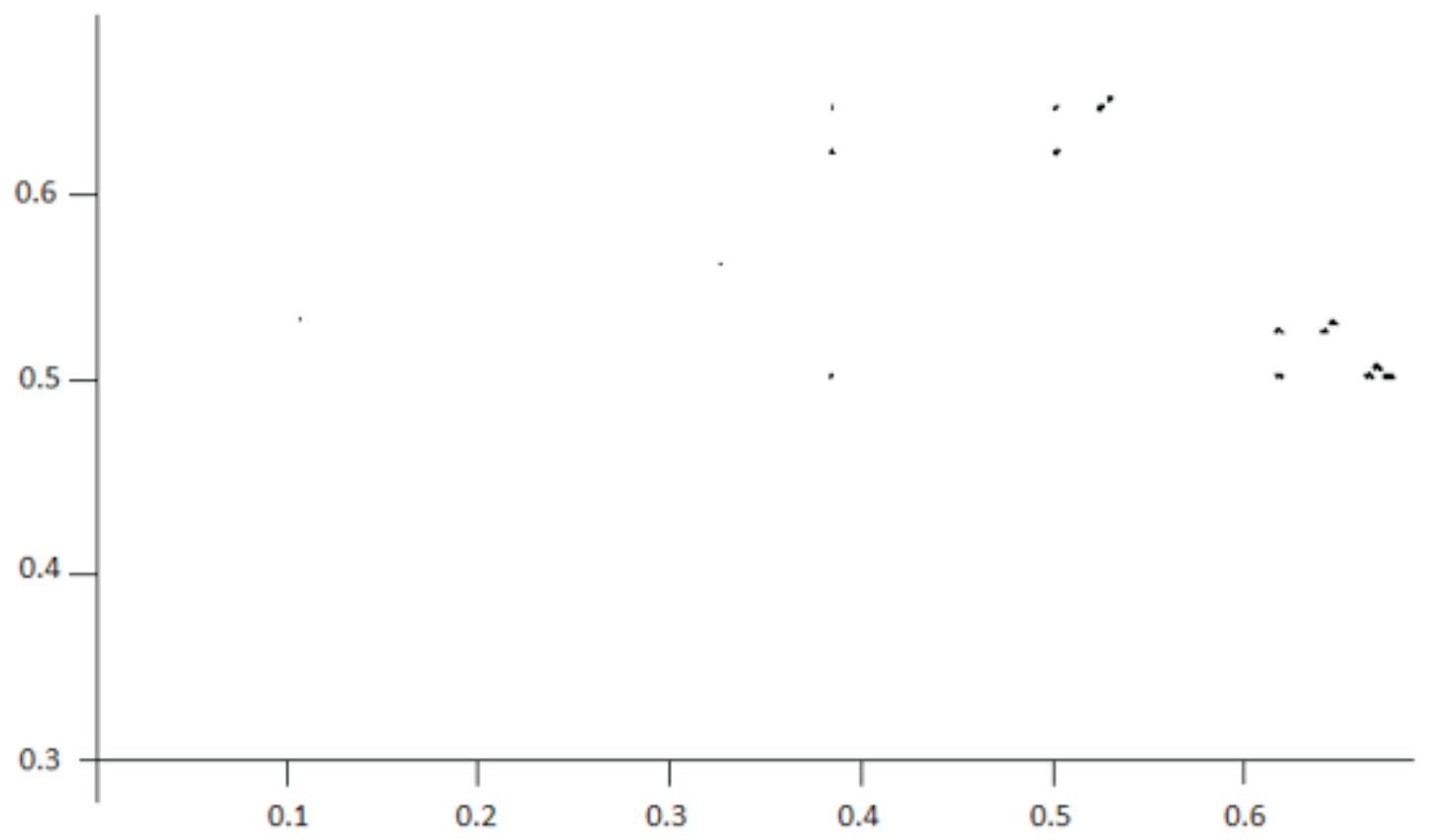

Figure 5

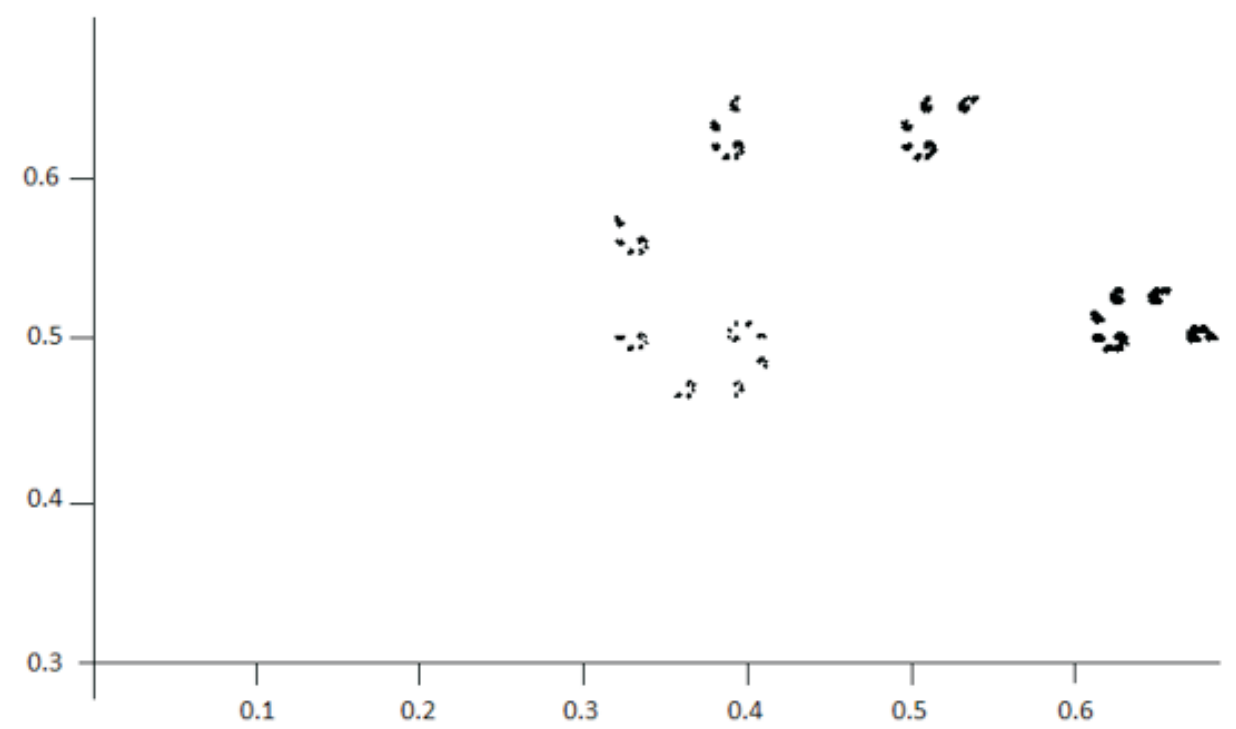

Figure 6 
Acknowledgements. The authors are very grateful to the reviewers whose extremely generous and valuable remarks and comments brought substantial improvements to the paper.

\section{References}

[1] F. Browder, On the convergence of successive approximations for nonlinear functional equations, Indag. Math., 30 (1968), 27-35.

[2] J.E. Hutchinson, Fractals and self similarity, Indiana Univ. Math. J., 30 (1981), 713-747.

[3] P. Jaros, Ł. Maślanka and F. Strobin, Algorithms generating images of attractors of generalized iterated function systems, Numer. Algorithms, 73 (2016), 477-499.

[4] Ł. Maślanka, F. Strobin, On generalized iterated function systems defined on $l^{\infty}$-sum of a metric space, J. Math. Anal. Appl., 461 (2018), 1795-1832.

[5] J. Matkowski, Integrable solutions of functional equations, Diss. Math., 127 (1975), $68 \mathrm{pp}$.

[6] R. Miculescu, Generalized iterated function systems with place dependent probabilities, Acta Appl. Math., 130 (2014), 135-150.

[7] A. Mihail and R. Miculescu, Applications of Fixed Point Theorems in the Theory of Generalized IFS, Fixed Point Theory Appl. Volume 2008, Article ID 312876, 11 pages doi: 10.1155/2008/312876.

[8] A. Mihail and R. Miculescu, A generalization of the Hutchinson measure, Mediterr. J. Math., 6 (2009), 203-213.

[9] A. Mihail and R. Miculescu, Generalized IFSs on Noncompact Spaces, Fixed Point Theory Appl. Volume 2010, Article ID 584215, 11 pages doi: $10.1155 / 2010 / 584215$.

[10] E. Oliveira, The Ergodic Theorem for a new kind of attractor of a GIFS, Chaos Solitons Fractals, 98 (2017), 63-71.

[11] E. Oliveira and F. Strobin, Fuzzy attractors appearing from GIFZS, Fuzzy Sets Syst., 331 (2018), 131-156.

[12] N.A. Secelean, Invariant measure associated with a generalized countable iterated function system, Mediterr. J. Math., 11 (2014), 361-372.

[13] N.A. Secelean, Generalized iterated function systems on the space $l^{\infty}(X)$, J. Math. Anal. Appl., 410 (2014), 847-858.

[14] F. Strobin, Attractors of generalized IFSs that are not attractors of IFSs, J. Math. Anal. Appl., 422 (2015), 99-108. 
[15] F. Strobin and J. Swaczyna, On a certain generalisation of the iterated function system, Bull. Aust. Math. Soc., 87 (2013), 37-54.

[16] F. Strobin and J. Swaczyna, A code space for a generalized IFS, Fixed Point Theory, 17 (2016), 477-493.

\section{Radu MICULESCU}

Faculty of Mathematics and Computer Science

Transilvania University of Braşov

Iuliu Maniu Street, nr. 50, 500091

Braşov, Romania

E-mail: radu.miculescu@unitbv.ro

Alexandru MIHAIL

Faculty of Mathematics and Computer Science

University of Bucharest, Romania

Academiei Street 14, 010014, Bucharest, Romania

E-mail: mihail_alex@yahoo.com

Silviu-Aurelian URZICEANU

Faculty of Mathematics and Computer Science

University of Piteşti, Romania

Târgul din Vale 1, 110040, Piteşti, Argeş, Romania

E-mail: fmi_silviu@yahoo.com 